\title{
Accuracy of Synovial Leukocyte and Polymorphonuclear Cell Count in Patients with Shoulder Prosthetic Joint Infection
}

\author{
Carol Strahm ${ }^{1 凶}$, Vilijam Zdravkovic², Claus Egidy², Bernhard Jost ${ }^{2}$ \\ 1. Division of Infectious Diseases and Hospital Epidemiology, Cantonal Hospital St. Gallen, St. Gallen, Switzerland \\ 2. Department of Orthopaedics and Traumatology, Cantonal Hospital St. Gallen, St. Gallen, Switzerland \\ $\square$ Corresponding author: Dr. Carol Strahm, Division of Infectious Diseases and Hospital Epidemiology, Cantonal Hospital St. Gallen, Rorschacher Str. 95, 9007 \\ St. Gallen, Switzerland. Email: carol.strahm@kssg.ch; Phone: +41 71494 2633; Fax: +41 714946339 \\ (C) Ivyspring International Publisher. This is an open access article distributed under the terms of the Creative Commons Attribution (CC BY-NC) license \\ (https://creativecommons.org/licenses/by-nc/4.0/). See http://ivyspring.com/terms for full terms and conditions.
}

Received: 2018.08.06; Accepted: 2018.09.14; Published: 2018.11.11

\begin{abstract}
Joint aspiration in suspected infected implants is a validated diagnostic method in establishing the diagnosis of prosthetic joint infection (PJI). Cut-off values for synovial leukocyte counts and differentials are well described for patients with hip and knee PJI. In 19 failed shoulder implants, a leucocyte count of $>12.2 \mathrm{G} / \mathrm{L}$ had a sensitivity and specificity of $92 \%$ and $100 \%$ respectively; A differential of $>54 \%$ neutrophils had a sensitivity of $100 \%$ and a specificity of $75 \%$.
\end{abstract}

Key words: prosthetic joint infection, shoulder arthroplasty, synovial fluid, cell count

Infection following shoulder arthroplasty is a rare but devastating complication. The rate of prosthetic joint infection (PJI) varies between $0.6 \%$ and $1.46 \%[1,2]$. The diagnosis of PJI in patients with shoulder arthroplasty is challenging. The diagnosis is established using a combination of clinical findings and laboratory studies[3]. Among these, analysis of synovial fluid cell count is a valuable adjunctive test for establishing the diagnosis of PJI[4, 5]. The optimal cut-off of synovial fluid leukocyte count and differential of polymorphonuclear leukocytes (PMN) is dependent on the duration of the joint life, site of the prosthesis, and presence of other comorbid conditions[3]. We herein present a study assessing the utility of synovial fluid cell count and differential in the diagnosis of patients with suspected shoulder PJI.

The Cantonal Hospital St. Gallen serves as a tertiary care centre for orthopaedic and trauma surgery in Switzerland. In patients with painful shoulder arthroplasties and planned revision surgery, we routinely perform pre-operative joint aspiration of synovial fluid assessing cell count, differential cell analysis, crystals and microbiological culture incubated for 14 days. Cell counts are performed by automated counting (Sysmex XN-1000, Sysmex Suisse AG, Horgen, Switzerland). In this study, we analysed the results of consecutive patients who underwent joint aspirations from April 2011 to June 2018. PJI was defined according the previously published IDSA criteria[5]. Due to a reported low specificity in the early postoperative phase, only joint aspirations performed $>6$ weeks after surgical intervention (i.e.; implantation or revision) were included in this study[6]. Informed consent was obtained from all patients enrolled in this study.

During the study period, 39 joint aspirations in patients with symptomatic shoulder arthroplasties were performed. Of these, 20 were excluded (3 punctures within six weeks of last surgical intervention, 11 cases with dry tap, 5 cases with microbiologic cultures [without cell count analyses], and in one case the results were inconclusive). Hence, the analysis included 19. In 16 of them, a leukocyte differential analysis was also performed. 13 cases met 
the PJI definition and six cases were classified as non-PJI.

The isolated organisms in monomicrobial infections included Cutibacterium acnes (5 cases), coagulasenegative staphylococci (3 cases), Staphylococcus aureus, Streptococcus anginosus and Finegoldia magna (one case each). Two patients had polymicrobial infections (table 1). Cell count in shoulder PJI ranged between 3.7 to $218.5 \mathrm{G} / \mathrm{L}$, and the percent of PMNs ranged between $74 \%$ to $99 \%$; In non-PJI cases the same ranged from $<0.1$ to $6.8 \mathrm{G} / \mathrm{L}$, and from 9 to $91 \%$ respectively. One patient in the non-PJI group had a high cell count and differential (6.8 G/L and 91\% PMN). In this case, foreign body reaction with polyethylene particles was documented on histologic examination of peri- prosthetic tissue. In the other 5 non-PJI cases, the cell count varied between $<0.1$ to $3.1 \mathrm{G} / \mathrm{L}$, and the PMNs percentage from 9 to $33 \%$. The 2 patients diagnosed with polymicrobial infections had high cell counts (>100 G/L).

To assess the optimal sensitivity and specificity of synovial fluid leukocyte count and PMN for differentiating aseptic failure from PJI, ROC curves were performed (figure 1). The area under the ROC curve was 0.987 for leukocyte count and 0.854 for PMN differential count. Optimal cut-off values for the 19 cases was $12.2 \mathrm{G} / \mathrm{L}$ for leukocyte counts (sensitivity $92 \%$ and specificity $100 \%$ ) and $54 \%$ for PMN differential (sensitivity $100 \%$ and specificity $75 \%$ ) (figure 1).

Table 1. Cell counts and differential PMN, culture results of 19 aspirates.

\begin{tabular}{|c|c|c|c|c|c|c|}
\hline $\begin{array}{l}\text { Cell count } \\
{[\mathrm{G} / \mathrm{L}]}\end{array}$ & $\% \mathrm{PMN}$ & Infection & Cultures & Interval from implantation & Revision arthroplasty? & $\begin{array}{l}\text { Interval from last surgery } \\
\text { (if not arthroplasty) }\end{array}$ \\
\hline 0.00 & $\mathrm{n} / \mathrm{a}$ & no & negative & 1.6 years & no & \\
\hline 0.38 & $33 \%$ & no & negative & 2.2 years & yes & \\
\hline 0.70 & $9 \%$ & no & negative & 2.2 years & no & \\
\hline 3.06 & $\mathrm{n} / \mathrm{a}$ & no & negative & 1.3 years & no & \\
\hline 3.10 & $7 \%$ & no & negative & 3.8 years & no & \\
\hline 3.71 & $74 \%$ & yes & C. acnes & 167 days & no & \\
\hline 6.83 & $91 \%$ & no & negative & 3 years & no & \\
\hline 17.51 & $95 \%$ & yes & CNS & 2.2 years & yes & \\
\hline 22.30 & $84 \%$ & yes & C. acnes & 50 days & yes & \\
\hline 36.50 & $95 \%$ & yes & C. acnes & 3.4 years & no & \\
\hline 37.40 & $80 \%$ & yes & F. magna & 1.1 years & no & \\
\hline 42.50 & $90 \%$ & yes & CNS & 5 years & yes & \\
\hline 46.35 & $92 \%$ & yes & CNS & 2 years & no & 315 days \\
\hline 54.67 & $88 \%$ & yes & S. anginosus & 105 days & yes & \\
\hline 74.39 & $\mathrm{n} / \mathrm{a}$ & yes & C. acnes & 1.3 years & no & \\
\hline 119.62 & $99 \%$ & yes & C. acnes / CNS & 7.8 years & no & 77 days \\
\hline 164.00 & $82 \%$ & yes & Staph aureus & 273 days & no & \\
\hline 176.95 & $94 \%$ & yes & C. acnes & 10.2 years & no & \\
\hline 218.50 & $89 \%$ & yes & C. avidum / CNS & 2.5 years & no & \\
\hline
\end{tabular}

Time interval from arthroplasty in years or days (when less than one year). When last revision was not the arthroplasty (2 patients), interval from last revision to puncture was specified separately (column "Interval from last surgery (if not arthroplasty)"). When puncture was performed in a revision arthroplasty (i.e. conversion from anatomic to inverse shoulder arthroplasty), this was specified in a separate column "Revision arthroplasty?".

CNS coagulase-negative staphylococcus; PMN polymorphonuclear leukocytes; $n$ /a not available
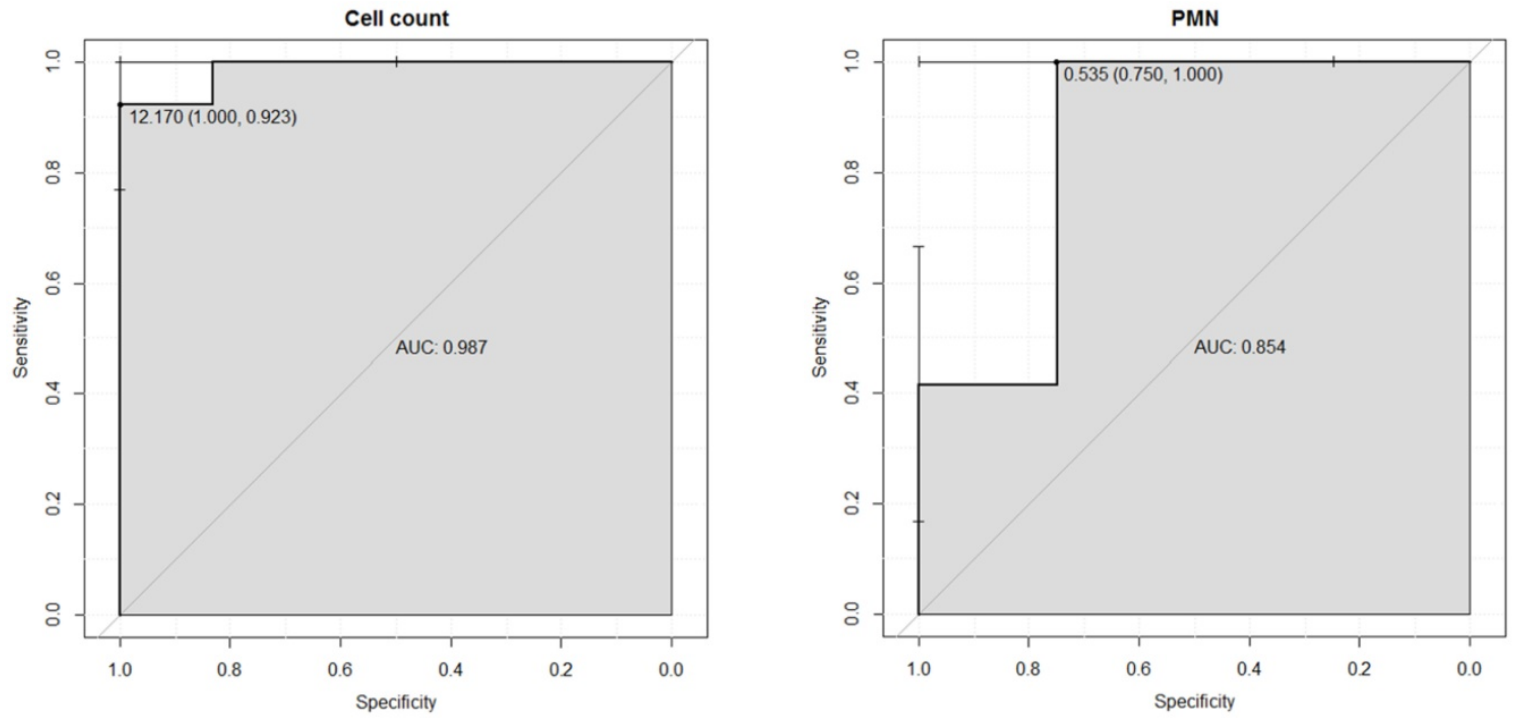

Figure 1. ROC curves for cell count and differential of polymorphonuclear leukocytes (PMN). 
Preoperative shoulder joint fluid aspiration assessing cell count and culture is a helpful diagnostic tool for establishing the diagnosis of PJI and - in culture positive cases - for guiding the choice of antimicrobial management in patients with hip and knee PJI[7]. In a recent meta-analysis, cut-off values ranged from 2.5 to $5 \mathrm{G} / \mathrm{L}$ and $60-89 \%$ for PMNs differential in total knee arthroplasty and total hip arthroplasty (THA)[8]. The results of this study support the hypothesis that cut-off values for hip or knee PJI cannot be extrapolated to other joints[5, 9]. The size of the joint, the surrounding synovial volume, the soft-tissue mantle, and the vascularization may influence the cut-offs of cell counts and differential in the diagnosis of PJI[9, 10]. On the basis of these arguments, it is conceivable that optimal cut-off values for shoulder PJI has not yet been optimally defined. To the best of our knowledge, only two studies have investigated the accuracy of synovial cell count in shoulder PJI. Piper et al.[11] analysed 28 patients (18 aseptic failure, 10 shoulder PJI) and used proposed cut-off values for knee PJI (>1.7 G/L and 65\% PMN). Only $5(50 \%)$ patients with confirmed PJI met the cell-count count criterion, and 7 (70\%) that for PMN[11]. Jerosch et al.[12] analysed 12 patients and suggested that a cell count above $30 \mathrm{G} / \mathrm{L}$ was suggestive for shoulder PJI. In the series published by Renz et al.[13], synovial fluid leukocyte count was available only for one patient with shoulder PJI. Infection was caused by $C$. acnes and synovial fluid analysis revealed a high cell count $(>80$ G/L) and differential (> $90 \%$ PMN). Our study includes 19 aspirates (6 aseptic failures, 13 shoulder PJI), and all cases with infection had values $\geq 3.7 \mathrm{G} / \mathrm{L}$ and $\geq 74 \%$ PMN. In contrast, only one case with aseptic loosening had a high cell count (case 7, table 1) likely due to a foreign body reaction. All three investigations have similar limitations, including a small sample size (28, 12 and 19 cases, respectively) and inherent limitations related to single-centre and retrospective study design. Nonetheless, they suggest that the cut-off used for synovial leukocyte value for establishing the diagnosis of shoulder PJI is higher than the ones described for knee or hip PJI[8, 12-15].

Cell count is an immune response associated with the microbial virulence of the infecting agent. One could hypothesize that in shoulder PJI, cell-counts are insensitive for diagnosis because low-grade microorganisms are commonly encountered in shoulder PJI[3, 11, 14]. The results of our study do not support this hypothesis. Low-grade infection organisms were associated with the presence of a high cell-count (table 1).

Of note, in $11(28 \%)$ of 39 cases, there was a dry tap. Synovial fluid aspiration from the shoulder joint is more challenging than the ones from knee joints. This may support the hypothesis that not all diagnostic concepts can be extrapolated from the knee joint to the shoulder joint.

In conclusion, synovial fluid aspiration in shoulder arthroplasty is a helpful preoperative diagnostic method in establishing the diagnosis of PJI. However, a high frequency of dry tap was observed. We found no indications that low-virulent organisms are associated with a low cell count in shoulder PJI. Our results and that of others[12, 13], indicate that the optimal synovial leukocyte and polymorphonuclear cell count cut-off for shoulder PJI is higher than the ones described for knee or hip PJI. Additional multi-centre studies are warranted to confirm those findings.

\section{Abbreviations}

PJI: prosthetic joint infection; PMN: polymorphonuclear leukocytes.

\section{Author Contributions}

Formal analysis: Carol Strahm, Vilijam Zdravkovic. Writing - original draft: Carol Strahm. Writing - review \& editing: Carol Strahm, Vilijam Zdravkovic, Claus Egidy, Bernhard Jost.

\section{Ethics/ Patient Consent}

Informed consent was obtained from patients. All procedures performed in studies involving human participants were in accordance with the ethical standards of the institutional and/or national research committee and with the 1964 Helsinki declaration and its later amendments or comparable ethical standards.

\section{Competing Interests}

The authors have declared that no competing interest exists.

\section{References}

1. Achermann Y, Sahin F, Schwyzer HK, Kolling C, Wust J, Vogt M. Characteristics and outcome of 16 periprosthetic shoulder joint infections. Infection. 2013; 41: 613-20.

2. Padegimas EM, Maltenfort M, Ramsey ML, Williams GR, Parvizi J, Namdari S. Periprosthetic shoulder infection in the United States: incidence and economic burden. J Shoulder Elbow Surg. 2015; 24: 741-6.

3. Tande AJ, Patel R. Prosthetic joint infection. Clin Microbiol Rev. 2014; 27: 302-45.

4. Lee YS, Koo KH, Kim HJ, Tian S, Kim TY, Maltenfort MG, et al. Synovial Fluid Biomarkers for the Diagnosis of Periprosthetic Joint Infection: A Systematic Review and Meta-Analysis. J Bone Joint Surg Am. 2017; 99: 2077-84.

5. Osmon DR, Berbari EF, Berendt AR, Lew D, Zimmerli W, Steckelberg JM, et al. Diagnosis and management of prosthetic joint infection: clinical practice guidelines by the Infectious Diseases Society of America. Clin Infect Dis. 2013; 56: e1-e25.

6. Christensen CP, Bedair H, Della Valle CJ, Parvizi J, Schurko B, Jacobs CA. The natural progression of synovial fluid white blood-cell counts and the percentage of polymorphonuclear cells after primary total knee arthroplasty: a multicenter study. The Journal of bone and joint surgery American volume. 2013; 95: 2081-7. 
7. Qu X, Zhai Z, Wu C, Jin F, Li H, Wang L, et al. Preoperative aspiration culture for preoperative diagnosis of infection in total hip or knee arthroplasty. J Clin Microbiol. 2013; 51: 3830-4.

8. Qu X, Zhai Z, Liu X, Li H, Wu C, Li Y, et al. Evaluation of white cell count and differential in synovial fluid for diagnosing infections after total hip or knee arthroplasty. PLoS One. 2014; 9: e84751.

9. Higuera CA, Zmistowski B, Malcom T, Barsoum WK, Sporer SM, Mommsen P, et al. Synovial Fluid Cell Count for Diagnosis of Chronic Periprosthetic Hip Infection. J Bone Joint Surg Am. 2017; 99: 753-9.

10. Ghanem E, Houssock C, Pulido L, Han S, Jaberi FM, Parvizi J. Determining "true" leukocytosis in bloody joint aspiration. J Arthroplasty. 2008; 23: 182-7.

11. Piper KE, Jacobson MJ, Cofield RH, Sperling JW, Sanchez-Sotelo J, Osmon DR, et al. Microbiologic diagnosis of prosthetic shoulder infection by use of implant sonication. J Clin Microbiol. 2009; 47: 1878-84.

12. Jerosch J, Schneppenheim M. Management of infected shoulder replacement. Arch Orthop Trauma Surg. 2003; 123: 209-14.

13. Renz N, Mudrovcic S, Perka C, Trampuz A. Orthopedic implant-associated infections caused by Cutibacterium spp. - A remaining diagnostic challenge. PLoS One. 2018; 13: e0202639.

14. Trampuz A, Hanssen AD, Osmon DR, Mandrekar J, Steckelberg JM, Patel R. Synovial fluid leukocyte count and differential for the diagnosis of prosthetic knee infection. Am J Med. 2004; 117: 556-62.

15. Schinsky MF, Della Valle CJ, Sporer SM, Paprosky WG. Perioperative testing for joint infection in patients undergoing revision total hip arthroplasty. J Bone Joint Surg Am. 2008; 90: 1869-75. 